\title{
A mathematical model for the dynamics of HIV/AIDS with gradual behaviour change
}

\author{
F. BARYARAMA, J. Y. T. MUGISHA* and L. S. LUBOOBI \\ Department of Mathematics, Makerere University, P.O. Box 7062, Kampala, Uganda
}

(Received 20 April 2006; revised 11 May 2006; in final form 5 June 2006)

\begin{abstract}
An HIV/AIDS model that incorporates gradual behaviour change is formulated with a variable force of infection for the adult population. The variability is modelled using a general function of time since introduction of the initial infective and exemplified for three specific functions. Expressions for the time taken for the reproductive number to reduce to unity and expressions for the time taken to attain a stationary steady state are deduced and discussed. Model projections for urban, peri-urban and rural Uganda are compared with corresponding antenatal clinic sites prevalence trends. The analysis shows that the dramatic decline in HIV prevalence in Uganda in the early 1990s was only possible through drastic declines in the force of infection. Since prevalence was high and reductions in frequency of sexual acts was minimal, the huge reduction could be attributed to reductions in probability of transmission per sexual act probably due to increased selective condom use among high risk sexual partnerships since overall condom use was low.
\end{abstract}

Keywords: Behaviour change; Variable force of infection; Replacement number; Antenatal

\section{Introduction}

Behaviour change is believed to be the main weapon against HIV/AIDS worldwide. A model by Ying-Yen and Cooke [1] on behaviour change and treatment of core groups and its effect on the spread of HIV/AIDS showed that behaviour change and treatment can eradicate the disease. In Uganda, many studies have reported declines in numbers of sexual partners and in frequency of sexual contacts as well as increase in condom use especially with non-steady or casual partners and among the youth [2]. Whereas it is known that both reductions in sexual activity and increase in condom use contributed toward the HIV prevalence declines in Uganda, there is still disagreement on which of these factors contributed most to the decline.

Factors that change sexual behaviour are subject to several constraints and limitations. Condoms do not offer $100 \%$ protection against HIV infection though their effectiveness, breakage, acceptablity and efficacy have been found at acceptable levels [3-7]. The general consesus is an estimated condom effectiveness of 60 to $95 \%$ with a mean of $87 \%$. On changes in fertility, there is compelling evidence that HIV/AIDS lowers fertility rates especially among those infected with HIV [8]. Carpenter et al. [9] from a population based cohort of over 3500 women aged 15-49 years in rural Uganda found lower fertility in HIV positive women

*Corresponding author. Email: jytmugisha@math.mak.ac.ug 
equal to three quarters that of uninfected women. Earlier fears were in the opposite direction that if a person learns of her HIV positive status, she would try to have children as quickly as possible [10]. On changes in sexually transmitted diseases (STDs), the presence of STDs is known to increase risks for HIV transmission as found by Wawer et al. [11] in a randomized community trial in Mwanza. Also Orroth et al. [12] found that a substantial proportion of new HIV infections in men in the non-intervention group were attributable to STDs.

Fortunately, a number of interventions have been proven to be particularly successful in imparting behaviour change. Among them is voluntary counselling and testing (VCT) that has been found to be feasible in most settings [13-15]. VCT has also been shown to be costeffective in terms of the number of new infections averted [16,17]. A recent study in the USA has verified a near to zero incidence among persons who had VCT, a 2-4\% among persons that had testing only and $11 \%$ for persons that were unaware of their HIV status [18]. However in some settings, readiness to VCT is initially low [19] and benefits have been reported as minimal [20].

In Uganda, these and other interventions led to declines in multiple partners from $39 \%$ in 1989 to $20 \%$ in 1995 and $11 \%$ in 2000 among males and from $18 \%$ in 1989 to $8 \%$ in 1995 and to $2.5 \%$ in 2000 among females [2]. Gray et al. [21], from a study of 174 monogamous HIV discordant couples in a population cohort in Rakai district, using data collected between November 1994 and October 1998, found a probability of transmission per sexual act of 0.001 and a frequency of sexual intercourse of 8.9 per month. According to Gray et al., the force of infection, $\beta c$ had declined from 0.433 at the beginnng of the epidemic [22], to 0.11, i.e. $0.001 \times 8.9 \times 12$ months by $1994-98$. Several other studies have attributed declines in HIV prevalence to changes in number of sexual partners and condom use [23-25]. Hence, variations in behaviour change parameters are the most significant to consider in the case of Uganda, compared to changes in progression, recruitment or removal rates.

In this paper, an HIV/AIDS model that incorporates gradual behaviour change is formulated with variable force of infection for the adult population. The variable force of infection is examined using three decreasing functions: linear, exponential and a lower positive bounded function. The model is analysed for conditions for attainment of a nontrivial disease-free equilibrium, time taken for the replacement number to reduce to one and the conditions for the attainment of an endemic equilibrium state with each of the three functions. The models are then applied to parameter values in the case of Uganda and a comparison of model projections for urban, semi-urban and rural Uganda made against HIV prevalence trends from corresponding antenatal clinic data.

\section{Model formulation}

Let an adult population comprise of susceptibles $S(t)$, infectives $I(t)$ and AIDS symptomatic cases $A(t)$. Define the following parameters as below:

- $p$ : the proportion of new borns that survive to adulthood;

- $a$ : mean age of first sex debut;

- $\lambda$ : the percapita birth rate;

- $\epsilon$ : the proportion of children born to infected mothers that are HIV-free;

- $\beta(t)$ : variable probability of HIV transmission per sexual act between a susceptible and an infective;

- $c(t)$ : variable average number of sexual acts per year; 
- $\omega(t)=\beta(t) c(t)$ : the variable force of infection;

- $\nu$ : the rate of progression from time of acquiring infection to development of AIDS symptoms (incubation period);

- $\mu$ : the natural mortarity rate of adults;

- $\tau$ : removal rate of AIDS cases (from development of AIDS symptoms to death);

- $\mu_{1}$ : the natural mortality rates of children aged 0 to $a$ years; and

- $p=\mathrm{e}^{\mu_{1}} a$ : survival to age $a$.

\subsection{The equations of the model}

The total population is given by $N(t)=S(t)+I(t)$ with the number of AIDS cases assumed removed from the population. Susceptibles are recruited through new borns that survive to adulthood. Susceptibles are removed by natural death $(\mu)$ and through those that acquire the HIV infection and hence join the infective class. The infectives are removed at an HIV accelerated fixed rate of $\nu+\mu$ and AIDS cases are removed at an AIDS accelerated fixed rate $\tau+\mu$. Time $t$ whenever used refers to time since the beginning of the epidemic. These assumptions lead to the following system of equations:

$$
\begin{gathered}
\frac{\mathrm{d} S}{\mathrm{~d} t}=p \lambda[\epsilon I(t-a)+S(t-a)]-\frac{\omega(t) S I}{N}-\mu S \\
\frac{\mathrm{d} I}{\mathrm{~d} t}=\frac{\omega(t) S I}{N}-(\nu+\mu) I \\
\frac{\mathrm{d} A}{\mathrm{~d} t}=\nu I-(\tau+\mu) A
\end{gathered}
$$

\subsection{The variable force of infection: $\omega(t)=\beta(t) c(t)$}

Using variable $\omega(t)=\beta(t) c(t)$ instead of constant $\beta c$ implies that $\omega_{0}=\beta_{0} c_{0}$ where $\beta_{0}=\beta(0)$ and $c_{0}=c(0)$. This formulation allows for incorporation of behaviour change from behavioural surveillance data, whenever available. However, the formulation requires specific functions to be used to describe $\omega(t)$. Three possible functions are used to model $\omega(t)$. Each is a decreasing function since positive behaviour change reduce both the probability of transmission, $\beta$ and the contact rate, $c$.

One, the function $\omega(t)$ could be a linear decreasing function defined as $\omega(t)=\omega_{0}(1-\alpha t), \alpha$ a constant where $\alpha, 0<\alpha<1$ is the annual rate of decline. Two, $\omega(t)$ could be an exponential decreasing function of the form $\omega(t)=\omega_{0}(1-\alpha)^{t}$. Both forms allow assessing the effect of changes in $\beta(t)$ and in $c(t)$ independent of each other or their joint effect, i.e. we could use $\omega(t)=\beta_{0} c_{0}(1-\gamma)^{t}(1-\delta)^{t}$ where $\gamma$ and $\delta$ are annual rates of change in $\beta$ and $c$, respectively. The disadvantage with these functions is that they each approach zero. It is hence reasonable to assume that reductions in sexual activity and in transmission rates per sexual contact may be quite substantial but remain well above zero long after persistent successful interventions.

Hence, we formulate a decreasing function with a positive lower bound of the form $\omega(t)=1 /\left(\kappa_{1}-\kappa_{2}(1-\alpha)^{t}\right)$ where $\kappa_{1}>\kappa_{2}>0$ and $0<\alpha<1$, with the following properties: 
1. $\omega(0)=\beta_{0} c_{0}=1 /\left(\kappa_{1}-\kappa_{2}\right), \kappa_{1}>\kappa_{2}$;

2. $\omega(t)$ tends to $1 / \kappa_{1}$ as $t$ approaches infinity. This is the positive lower bound;

3. $\omega(t)$ is a monotonically decreasing function from $1 /\left(\kappa_{1}-\kappa_{2}\right)$ at $t=0$ to $1 / \kappa_{1}$ as $t$ tends to positive infinity;

4. the ratio $\kappa_{1} /\left(\kappa_{1}-\kappa_{2}\right)$ represents the number of times $\beta_{0} c_{0}$ decreases after a long time from the start of interventions;

5. the values of $\omega(0)$ and $\omega(\infty)$ are independent of the annual rate of change, $\alpha$. Hence for the same $\omega(0)$ and $\omega(\infty)$, there exists different curves of $\omega(t)$ corresponding to different values of the rate of change, $\alpha$. Small values of $\alpha$ are associated with large values of $\omega(t)$ implying slow declines in high risk sexual behaviours; and

6. suitable choices of $\omega(0)$ and $\omega(\propto)$ uniquely determine the values of $\kappa_{1}$ and $\kappa_{2}$ independent of annual rate of decline, $\alpha$.

From the above, it follows that $\kappa_{1}=1 / \omega(\propto)$ and $\kappa_{2}=(1 / \omega(\propto))-(1 / \omega(0))$.

\subsection{Comparison of the three functions}

To illustrate the three functions, let $\omega(t)=0.4$ at the beginning of the epidemic and allow $\omega(t)$ decrease over a period of 30 years to stablize at 0.1 , i.e. $\omega(30)=0.1$. The linear, exponential and lower bounded functions to achieve this are plotted on the same axes as shown on figure 1.

The linear function is derived by finding an annual rate of decline which is given by $0.4(1-30 \alpha)=0.1$. This gives $\alpha=0.025$. Hence, the linear function is $\omega(t)=0.4(1-0.025 t)$. The linear function becomes zero at $t=40$ years. This makes it unrealistic for long term projections.

In the case of the exponential decline, the annual rate of decline is derived from $0.4(1-\alpha)^{30}=0.1$. Solving gives $\alpha=0.045$ and hence the exponential function is $\omega(t)=0.4 * 0.955^{t}$. This function comes unrealistically close to zero. For example, at $t=60$ years, $\omega(t)=0.025$. Since observed contact rates are well over 100 contacts per year, this gives a probability of transmission per contact of 0.00025 , far below any documented observed rates. Hence, this function cannot be used for long term projections.

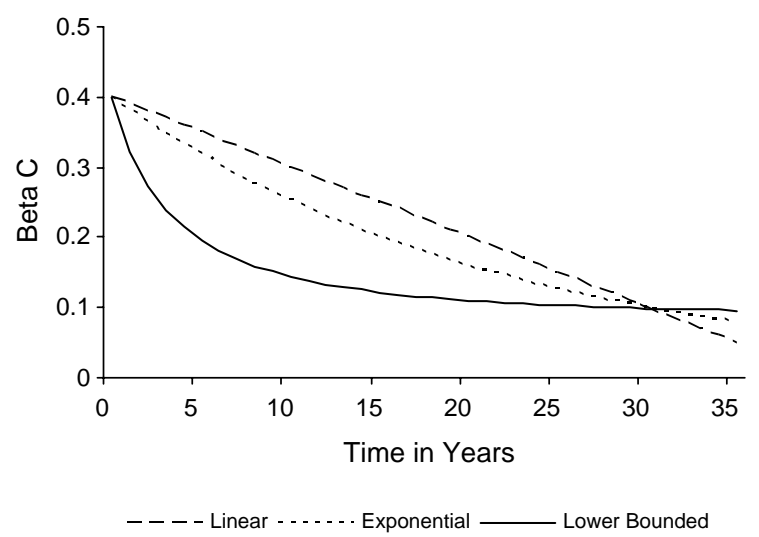

Figure 1. Comparison of the linear, exponential and lower bounded forms of $\omega(t)$ showing a decline from 0.4 to 0.1 in 30 years. 
In the case of the lower bounded function, set $\omega(\infty)$ to slightly less than 0.1 at 0.09 . From $\omega(\infty)=1 / k_{1}$, we get $k_{1}=11.1$. But from $\omega(0)=1 / k_{1}-k_{2}$, we get $11.1-k_{2}=$ $1 / 0.4=2.5$.

Hence $k_{2}=8.6$. It follows that $\omega(t)=1 /\left(11.1-8.6(1-\alpha)^{t}\right)$. It is important to note that for any selected $\alpha$, this function reduces $\beta c$ from 0.4 to asymptotically approach the value of 0.09 . However, for small values of $\alpha$, such a reduction takes many years. Setting $1 /\left(11.1-8.6(1-\alpha)^{30}=0.1\right)$ gives $\alpha=0.07$. Hence, a possible lower bounded function is $1 /\left(11.1-8.6 * 0.93^{t}\right)$. This lower bounded function is realistic in situations where the initial interventions are concentrated to cause huge positive behaviour change that result in large initial declines in $\beta c$. The limiting value of 0.09 at a contact rate of 100 contacts per year gives a probability of transmission per contact of 0.0009 which is close to the rates observed in rural Uganda in the mid to late 1990s [21].

\section{Analysis of the model}

The analysis is restricted to finding the conditions for attainment of the non-trivial diseasefree equilibrium and hence conditions for eradication of the disease, the time taken for the replacement number $R(t)$ to reduce to unity denoted by $t^{\prime}$ and the time taken to attain the endemic equilibrium state denoted by $t^{*}$. The replacement number $R(t)$ is the number of infections by one infective during his/her entire infectious period at any time $t$ of the epidemic. When the replacement number reduces and reaches a critical value of one, then each infective only manages to replace himself or herself. Further reduction of the replacement number to less than one keeps the epidemic under control and if sustained, moves the epidemic to extinction. By this definition, the replacement number differs from the reproduction number $R_{0}$ which is the number of new infections attributed to one infective if introduced in a disease-free community. The endemic equilibrium state is attained when the time rates of change of susceptibles and infectives are simultaneously equal to zero with a positive number of infectives. Usually, this corresponds to susceptibles and infectives attaining constant population sizes of $S *$ and $I *$ respectively at a time beyond attainment of the peak prevalence.

\subsection{Attaining the non-trivial disease-free equilibrium}

The non-trivial disease-free equilibrium corresponds to $\mathrm{d} S / \mathrm{d} t=(p \lambda-\mu) S=0, S>0$, $I=0$ as $\mathrm{t} \rightarrow \infty$ and is attained when $p \lambda=\mu$. But since $p=\mathrm{e}^{-\mu_{1} a}$, it follows that attainment of a non-trivial disease-free equilibrium depends on the birth rate $\lambda$, the age of first sex $a$ and the mortality rates $\mu$ and $\mu_{1}$. Attainment of a non-trivial disease-free equilibrium is however independent of the behaviour change parameter $\omega(t)$. Figure 2 shows the age of first sex debut required to satisfy the non-trivial disease-free condition for $\mu=0.02$ and $\mu_{1}=0.03$, rates that apply to the Ugandan situation [22]. The age of first sex to meet the non-trivial disease free condition is 31 years when $\lambda=0.05$ ! However, a reduction in the per capita birth rate from 0.05 to 0.035 (without reductions in mortality rates) lowers the mean age of first sex debut needed to attain a non-trivial disease-free condition to 19 years. To attain disease-free equilibrium and hence eliminate the disease when the age of first sex is 15 years requires lowering the crude birth rate to about $3 \%$ per annum if the crude death rates, $\mu$ and $\mu_{1}$ remain unchanged. These findings suggest that family planning interventions may have a significant role to play in the eradiction of the HIV/AIDS epidemic. 


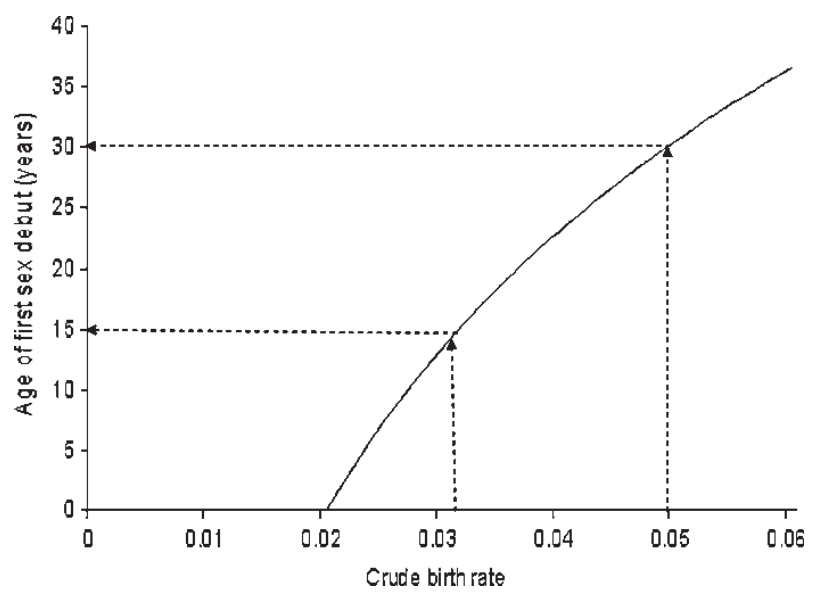

Figure 2. Birth rate versus age of first sex debut required to attain the non-trivial-disease equilibrium $\left(a=[-\ln (\mu / \lambda)] / \mu_{1}, \mu_{1}=0.03, \mu=0.02\right)$.

However, both $\mu$ and $\mu_{1}$ have an opposing role. Lower mortality rates push the required age of first sex to attain a disease-free equilibrium to higher ages and vice versa. If birth rates are fixed at 5\% and under-fifteen mortality rate at 3\%, the disease-free equilibrium can be attained at the age of first sex of 15 years if the adult mortality rate is (figure $3 a$ ). This is clearly undesirable. Likewise, lowering under-fifteen mortality rates when the crude birth rate is fixed at $5 \%$, adult mortality rate fixed at $2 \%$ gives no hope of ever eradicating the disease (figure 3b). From public health considerations, attaining the disease-free equilibrium can be achieved by lowering the crude birth rate plus other non-behaviour interventions.

\subsection{Time for replacement number to reduce to one}

Using the replacement number $R(t)=\omega(t) /(\nu+\mu)$ and substituting for specific forms of $\omega(t)$ gives expressions for the time taken $\left(t^{\prime}\right)$ for the replacement number to reduce to one (i.e. $R(t)=1)$. For the linear form, solving $\omega_{0}\left(1-\alpha t^{\prime}\right) /(\nu+\mu)=1$ gives $t^{\prime}=\omega_{0}$ $-(\nu+\mu) / \omega_{0} \alpha$. Similarly, solving the corresponding exponential and lower bounded functions gives expressions of $t^{\prime}$ given in table 1 .

3.2.1 The endemic equilibrium. The endemic equilibrium is attained at a non-zero HIV prevalence with positive numbers of susceptibles and infectives. Assuming that the positive stationary state is realised at time $t=t^{*}$, then according to equation (2)

$$
\frac{S^{*}}{N^{*}}=\frac{\nu+\mu}{\omega(t *)}
$$

where $S *=S(t *)$ and $N *=N(t *) . I *$ is similarly defined as $I *=I(t *)$. From equation (1) subject to equation (4) and noting that $I\left(t^{*}-a\right) \simeq I\left(t^{*}\right)$ and $A\left(t^{*}-a\right) \simeq A\left(t^{*}\right)$

$$
p \lambda\left(\epsilon I\left(t^{*}\right)+S(t *)\right)-\omega(t *) \frac{I\left(t^{*}\right) S\left(t^{*}\right)}{N\left(t^{*}\right)}-\mu S\left(t^{*}\right)=0
$$




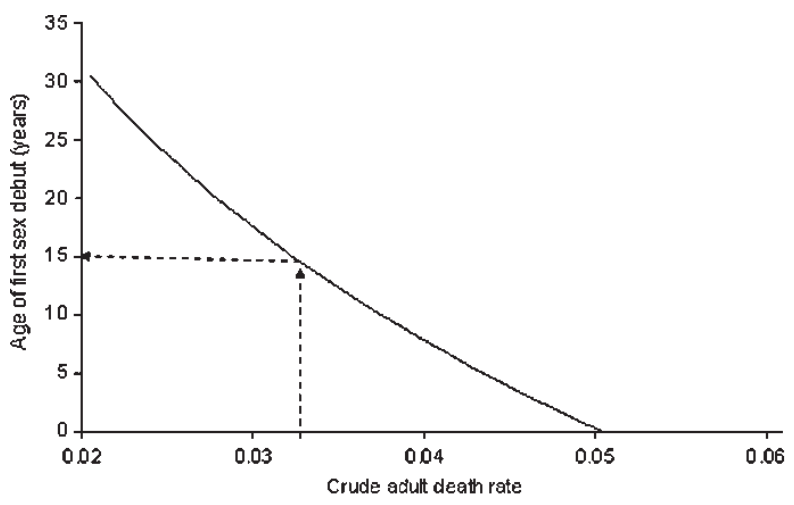

(a) Required age of first sex by crude adult death rate

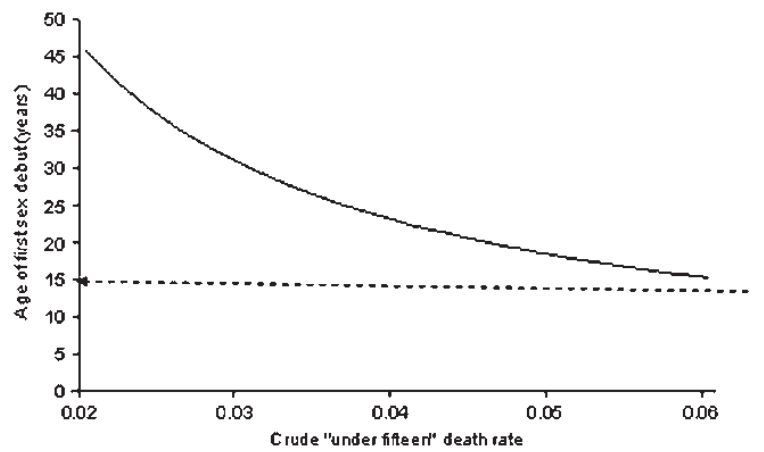

(b) Required age of first sex by crude "under fifteen" death rate

Figure 3. Death rates versus age of first sex debut required to attain the non-trivial-disease equilibrium $\left(a=(-\ln (\mu / \lambda)) / \mu_{1}\right.$, fixed values are: $\left.\mu_{1}=0.03, \mu=0.02, \lambda=0.05\right)$.

Dividing by $N\left(t^{*}\right)$ and substituting with $S(t *) / N\left(t^{*}\right)=(\nu+\mu) / \omega\left(t^{*}\right)$ and $I\left(t^{*}\right) / N\left(t^{*}\right)=$ $1-\left((\nu+\mu) / \omega\left(t^{*}\right)\right)$ leads to equation (6)

$$
p \lambda\left[\epsilon\left(1-\frac{\nu+\mu}{\omega\left(t^{*}\right)}\right)+\frac{\nu+\mu}{\omega\left(t^{*}\right)}\right]-\omega\left(t^{*}\right)\left[1-\frac{\nu+\mu}{\omega\left(t^{*}\right)}\right]\left[\frac{\nu+\mu}{\omega\left(t^{*}\right)}\right]-\mu \frac{\nu+\mu}{\omega\left(t^{*}\right)}=0
$$

Table 1. Expressions for time for replacement number to reduce to one, $t^{\prime}$ and time taken to reach endemic equilibrium, $t^{*}$.

\begin{tabular}{llll}
\hline & \multicolumn{3}{c}{ Form of $\omega(t)$} \\
\cline { 2 - 4 } & \multicolumn{1}{c}{ Linear } & Exponential & Lower bound $>0$ \\
\hline$\omega(t)$ & $\omega_{0}(1-\alpha t)$ & $\omega_{0}(1-\alpha)^{t}$ & $\frac{1}{\kappa_{1}-\kappa_{2}(1-\alpha)^{t}}$ \\
Time to $t^{\prime}$ & $\frac{\omega_{0}-(\nu+\mu)}{\omega_{0} \alpha}$ & $\frac{\ln \left(\frac{\nu+\mu}{\omega_{0}}\right)}{\ln (1-\alpha)}$ & $\left.\frac{1}{\kappa_{2}}\left(\kappa_{1}-\frac{1}{\nu+\mu}\right)\right)$ \\
Time to $t^{*}$ & $\frac{\omega_{0}-(\nu+\mu) q}{\omega_{0} \alpha}$ & $\frac{\ln \left(\frac{\nu+\mu}{\omega_{0}} q\right)}{\ln (1-\alpha)}$ & $\frac{\ln \left[\frac{1}{\kappa_{2}}\left(\kappa_{1}-\frac{1}{q(\nu-\mu}\right)\right]}{\ln (1-\alpha)}$ \\
\hline
\end{tabular}

Where $q=\frac{[p \lambda(1-t)+\nu]}{\nu+\mu-p \lambda \epsilon}$. 
which on simplification gives

$$
\omega\left(t^{*}\right)=\frac{(\nu+\mu)[p \lambda(1-\epsilon)+\nu]}{\nu+\mu-p \lambda \epsilon}
$$

Since $\omega(t) \geq 0$, we require that $\nu+\mu>p \lambda \epsilon$. Note that $\eta=p \lambda \epsilon /(\nu+\mu)$ is the number of children born by an infected mother during the period she is infected that survive the period $(0, a)$. Thus, $\nu+\mu>p \lambda \epsilon \Rightarrow \eta<1$ for the existence of a positive stationary state. This condition is readily satisfied when $p \lambda<\mu$ since $0<\epsilon<1$ and $\nu>0$.

Consider the expression $q=[p \lambda(1-\epsilon)+\nu] /(\nu+\mu-p \lambda \epsilon)$. The quantity $q$ exhibits threshold phenomenon with $q=1$ corresponding to $p \lambda=\mu$ when the attainment of the endemic equilibrium coincides with when the replacement number reduces to unity. Also $t^{*}>t^{\prime}$, when $q<1$ implying that $p \lambda<\mu$. This condition is facilitated by a number of factors that include: low crude birth rate, high age of first sexual debut, high adult mortality rate and high death rate among those not yet sexually active. With age of first sex at 15 years and an under-fifteen mortality rate of $3 \%$ and an adult mortality rate of $2 \%$, an endemic equilibrium can be attained if the crude birth rate is less or equal to $3.1 \%$. It is therefore unlikely that such an equilibrium may be attained in Uganda.

Using $\omega_{0}=0.4, \nu=0.125, \mu=0.02, \mu_{1}=0.03, \lambda=0.05, a=15$ and the values used to plot figure 1 gives $q=1.097$ and the times to $R(t)=1, t^{\prime}$ are 25.5, 22.0 and 9.9 years for linear, exponential and lower bounded functions, respectively. Corresponding times to an endemic equillibrim $t *$ are 28.0, 24.2 and 8.0 years.

The endemic equilibrium when $t^{*}<t^{\prime}$, as in the case of a lower bounded function, suggests that it would not exist. Figure 4 shows the two regions when recruitment into adulthood is less or greater than adult mortality.

The figure shows that if the changes in $\beta c$ are described by the lower bounded function, the replacement number reduces to unity much faster (in ten years) than for either the linear

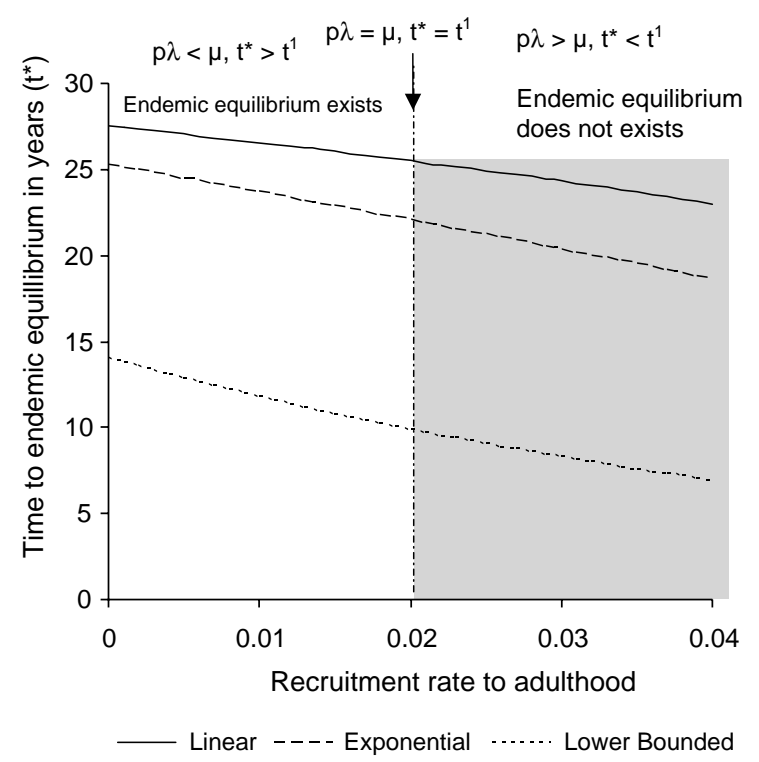

Figure 4. Time to endemic equilibrium (and to $R(t)=1$ when $p \lambda=\mu_{2}$ ) assuming interventions started at the begining of the epidemic. 
(in 26 years) or exponential (in 22 years) functions. Attainment of the endemic equilibrium though theoretically possible remains difficult to achieve since it requires that the recruitment rate into adulthood should be less than the removal rates of adults i.e. $p \lambda<\mu$. This is not possible for most communities especially in developing countries with high birth rates and overall net positive population explosion growth rates.

\section{Application of the model to the Ugandan situation}

Interventions against HIV in Uganda started in the late 1980s. We assume that interventions were intense and hence of the order of the lower bounded function earlier presented. Assuming stepwise functions (8) for urban and (9) for rural communities in Uganda gives figure 5 as the time trend of the behaviour change parameter. This time trend a slow linear decline from $\beta c=0.433$ in urban Uganda in 1980 upto 1987 and thereafter a huge lower bounded decline to $\beta c=0.1$ by 2000 . For rural areas, the time trend shows initial lower $\beta c=0.4$ in 1980 that showed no change upto 1989 followed by a huge lower bounded decline reducing the rural $\beta c$ to 0.1 by the year 2000. These time trends in $\beta c$ are consistent with available behavioural data on the HIV epidemic in Uganda over this time period $[21,22]$. They are also consistent with intervention efforts launched by local, national and international networks to fight the Ugandan HIV epidemic.

$$
\begin{gathered}
\omega_{u(t)}= \begin{cases}0.433(1-0.02 t), & t \leq 7 \\
\left(20-17.25 * 0.94^{(t-7)}\right)^{-1}, & t \geq 7\end{cases} \\
\omega_{r(t)}= \begin{cases}0.4, & t \leq 9 \\
\left(20-17.5 * 0.9^{(t-9)}\right)^{-1}, & t \geq 9\end{cases}
\end{gathered}
$$

Massive interventions were launched in the late 1980s starting from urban areas and quickly spreading out to rural areas. The effect of these massive interventions was drastic declines in $\beta c$. The impact of declines in $\beta c$ are illustrated by comparing model projections with data from three antenatal surveillance clinic sites; one urban ANC established in 1989 in Kampala, one semi-urban clinic established in the same year in Mbale town close to the Eastern boarder with Kenya and one rural site at Mutolore in Southwestern Uganda. Data for

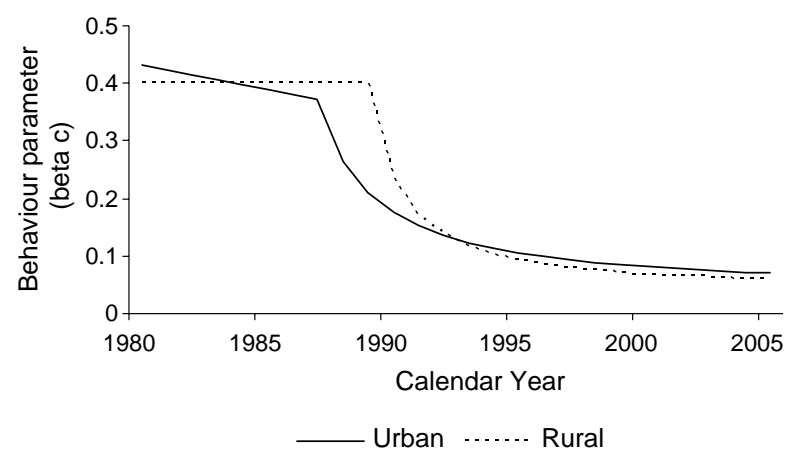

Figure 5. Form of behaviour parameter $(\beta c)$ over time: case for Uganda. 


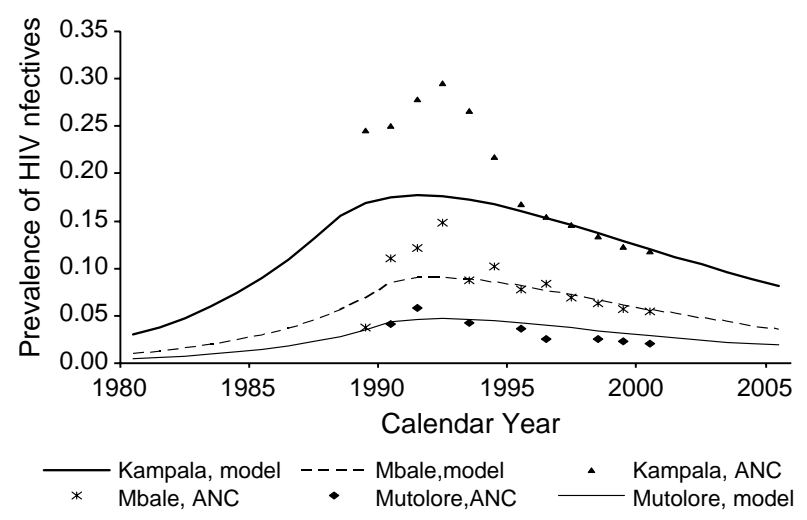

Figure 6. Rural and urban model prevalence of Infectives compared to ANC prevalence: case for Uganda.

Mutolere is available since 1990 with data for 1992, 1994 and 1997 not available. The initial prevalence in 1980 are set at 3\% for Kampala, 1\% for Mbale and $0.5 \%$ for Mutolore.

Drastic declines in $\beta c$ showed an immediate impact on the epidemic. As seen from figure 6, infection rates among pregnant women declined [2]. HIV infection rates of $30 \%$ in 1992 at Nsambya dropped to $8 \%$ in 2000 . However, our model fails to capture the observed high peak prevalence at Nsambya. Instead, the model gives a peak of $18 \%$ in 1991 . Similarly the high peak of $15 \%$ at Mbale is not captured by the model. Instead, the model gives a peak of $9 \%$ in 1992. For Mutolore, a rural site, the model and the ANC data show close agreement with a peak prevalence of $5 \%$ in 1992. Despite failing to reproduce the peak prevalence for the urban and semi-urban sites, the model gives a good fit to the data beyond the peak prevalence.

\section{Discussions}

Gradual positive behaviour change leading to persistent reduction in the force of infection by use of variable force of infection in HIV/AIDS modelling was presented and used to demonstrate trends in HIV prevalence in Uganda. This was verified for three ANC sites; an urban site, a peri-urban site and a rural site. The approach fairly explained trends in HIV prevalence in Uganda in the 1980s and 1990s. The models though did not reproduce the high peak prevalence observed around 1992 in major urban towns although the timing of the peak prevalence do coincide. This may suggest that the high peaks were a result of other factors, in addition to sexual behaviour, are responsible for the excess HIV prevalence. Such factors could include selective participation with high-risk pregnant women seeking antenatal clinic services or mobility of high-risk women to urban areas among others. On the other hand, the model may have failed to peak the high peak prevalence due to its simplicity. The epidemic could have first concentrated in a certain age group or social group. Such heterogeneity could be responsible for the observed high peaks.

The proposed lower bounded function captures the situation of massive interventions that results in a sharp decline in infection rates soon after the onset of the interventions. Such a situation cannot be captured by linear, or even exponential declines. The lower bounded function hence better describes the Uganda HIV epidemic that is characterised by massive interventions launched towards the end of the 1980s. 
Using the gradual behaviour change model, a decrease in the behaviour parameter directly leads to a decrease in the number of secondary infections. Expressions for the time taken for the number of secondary infections to reduce to 1 and the time taken to attain a positive stationary state were found to be related by a quantity that showed threshold phenomena with respect to a demographic equilibrium $\left(p \lambda=\mu_{2}\right)$. The findings reaffirm that HIV prevalence not only depends on the number of persons infected with HIV but also on the number of susceptibles. A reduction (or increase) in the number of susceptibles through recruitment (or death) does not affect the reproductive number or the incidence but increases (or reduces) the HIV prevalence. This reality is contrary to common belief that the incidence curve reaches its peak before the prevalence peaks and those that have observed the contrary attribute the contradiction to mortality effects and not to high recruitment. These results suggest that with Uganda's high fertility rate leading to high recruitment into the sexually active age group, it is possible that the peak prevalence in Uganda was attained before attaining substantial declines in HIV incidence in agreement with "high rate of replacement" explanation by Mulder [25]. The finding reaffirm the belief that prevalence trends are heavily dependant on the demographic dynamics of the population, and are of limited validity in evaluating the impact of HIV/AIDS interventions.

The model developed using different forms of $\omega(t)$ suggest that massive initial interventions are needed in order to contain the epidemic. Intervention impacts in the order of linear declines are not enough; impacts should be of the order of exponential declines or faster. The model highlights the importance of delayed age of first sex and birth rates. Older age of first sex and lower birth rates are independently desirable to achieve disease free equilibrium. But higher age of first sex and lower birth rates independently reduce the recruitment rate into the sexually active age group, which reduce the chances for attainment of a positive stationary state. The effect of an increase in age of first sex has not been well studied. Intuitively, sex at an older age means fewer individuals exposed and hence fewer infections. However, this also means fewer individuals engaged in reproduction, hence leading to fewer births and fewer adults. The interplay of this could be a sustained epidemic among those that are sexually active. This calls for further study.

The utility of this approach depends on the ability to get functions that best describe the behaviour parameter over time for a given setting. Our results show that the model failed to catch the high peaks observed in Uganda. This could be due to a number of factors. One, our one-stage model does not account for the various stages with differing infectious levels of infectiousness. Two, the model grouped all adults together and hence fails to capture age differentials. Three, the model rely on behaviour data and functions that may be difficult to get for a particular setting. The model proved not suitable for high prevalence settings but may be appropriate for low and medium prevalence epidemics.

\section{References}

[1] Ying-Yen, H. and Cooke, K., 2000, Behaviour change and treatment of core groups: its effect on the spread of HIV/AIDS, IMA Journal of Mathematics Applied in Medicine and Biology, 17, 213-241.

[2] UNAIDS/Uganda, 2000, Uganda epidemiological fact sheet on HIV/AIDS and sexually transmitted diseases. (Geniva: UNAIDS; DHS 1995, 2000/2001).

[3] Davis, K.R. and Weller, S.C., 1999, The effectiveness of condoms in reducing heterosexual transmission of HIV, Family Planning Perspectives, 31(6), 272-279.

[4] Macaluso, M., Kelagham, J. and Artiz, L., 1999, Mechanical failure of the latex condom in a cohort of women at high STD risk, Sexually Transmitted Diseases, 28(8), 450-458.

[5] Frezieres, R.G., Walsh, T.L. and Nelson, V.A., 1999, Evaluation of the efficacy of a polyurethane condom: a randomized, controlled study, Family Planning Perspectives, 31(2), 81-87. 
[6] Pinkerton, S.D. and Abramson, P.R., 1997, Effectiveness of condoms in preventing HIV transmission, Social Science and Medicine, 44, 1303-1312.

[7] Gordon, R., 1989, A critical review of the physics and statistics of condoms and their role in individuals versus societal survival of the AIDS epidemic, Journal of Sex and Marital Therapy, 15(1), 5-30.

[8] Gregson, S., Zhuwau, T., Anderson, R.M. and Chandiwana, S.K., 1997, HIV and fertility change in rural Zimbabwe, Health Transition Review, 7, 89-112.

[9] Carpenter, L.M., Kamali, A. and Ruberantwari, A., 1999, Rates of HIV-1 transmission within marriage in rural Uganda in relation to HIV sero-status of the partners, AIDS, 13, 1083-1089.

[10] Setel, P., 1995, The effects of HIV and AIDS on fertility in East and Central Africa, Health Transition Review, $\mathbf{5}, 179-189$.

[11] Wawer, M.J., Gray, R.H. and Sewankambo, N.K., 1998, A randomised, community trial of intensive sexually transmitted disease control for AIDS prevention, Rakai, Uganda, AIDS, 12, 1211-1225.

[12] Orroth, K.K., Gavyole, A. and Todd, J., 2000, Syndromic treatment of sexually transmitted dieases reduces the proportion of incident HIV infections attributable to these diseases in rural Tanzania, AIDS, 14, 1429-1437.

[13] Wilkinson, D., Wilkinson, N. and Lombard, C., 1997, On-site HIV testing in resource-poor settings: is one rapid test enough?, AIDS, 11, 377-381.

[14] Downing, R.G., Otten, R.N. and Marum, E., 1998, Optimizing the delivery of HIV counselling and testing services: the Uganda experience using rapid HIV antibody test algorithms, Journal of Acquired Immune Deficiency Syndromes and Human Retrovirology, 18, 384-388.

[15] Abouya, L., Coulibaly, I.M. and Wiktor, S.Z., 1998, The Cote d'Ivoire national HIV counselling and testing program for tuberculosis patients: implementation and analysis of epidemiological data, AIDS, 12, 505-512.

[16] Sweat, M., Gregorich, S., Sangiwa, G., Furlonge, C., Balmer, D. and Kamenga, C., 2000, Cost-effectiveness of voluntary HIV-1 counselling and testing in reducing sexaul transmission of HIV-1 in Kenya and Tanzania, Lancet, 356, 113-121.

[17] Coates, T.J., 2000, Efficacy of voluntary HIV-1 counselling and testing in individuals and couples in Kenya, Tanzania and Trinidad: a randomized trial, Lacent, 256, 103-112.

[18] Holtgrave, R. and Anderson, T., 2004, Utilizing HIV transmission rates to assist in pritizing HIV prevention services, Journal of STDS/AIDS, 15, 789-792.

[19] Fylkesnes, K., Haworth, A. and Rosensvard, C., 1999, HIV counselling and testing: overemphasizing high acceptance rates a threat to confidentiality and the right not to know, AIDS, 13, 2469-2474.

[20] Sahlu, T., Kassa, T. and Agonafer, T., 1999, Sexual behaviours, perception of risk of HIV infection, and factors associated with attending HIV post-test counselling in Ethiopia, AIDS, 13, 1263-1272.

[21] Gray, R.H., Wawer, M.J., Bookmeyer, R., Sewankambo, N.K., Serwadda, D. and Wabwire-Mangen, F., 2001, Probability of HIV-1 transmission per coital act in monogamous, heterosexual, HIV-1-discordant couples in Rakai, Uganda, Lacent, 357, 1149-1153.

[22] Mugisha, J.Y.T. and Luboobi, L.S., 2000, The endemicity of HIV/AIDS in a three-age structured population, IJOMAS, 16(2), 125-136.

[23] Ntozi, J.P.M. and Ahimbisibwe, F.E., 1999, Some factors in the decline of AIDS in Uganda, The Continuing African HIV/AIDS Epidemic, 1999, 93-107.

[24] Konde-Lule, J.K., Sewankambo, N. and Morris, M., 1997, Adolescent sexual networking and and HIV transmission in rural Uganda, Health Transition Review, 7, 89-100.

[25] Mulder, D., Nunn, A., Kamali, A. and Kengeya-Kayondo, J., 1995, Decreasing HIV-1 seroprevalence in young adults in rural Uganda cohort, BMJ, 311, 833-836. 


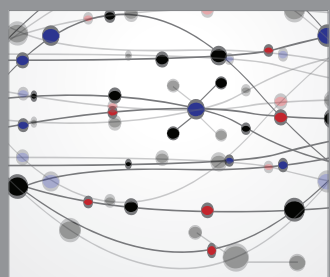

The Scientific World Journal
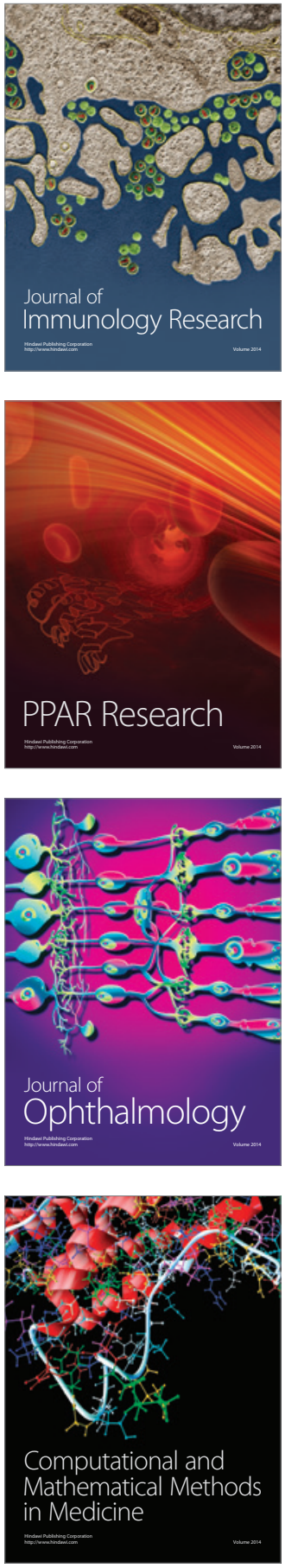

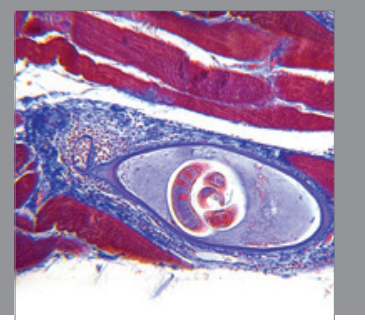

Gastroenterology

Research and Practice
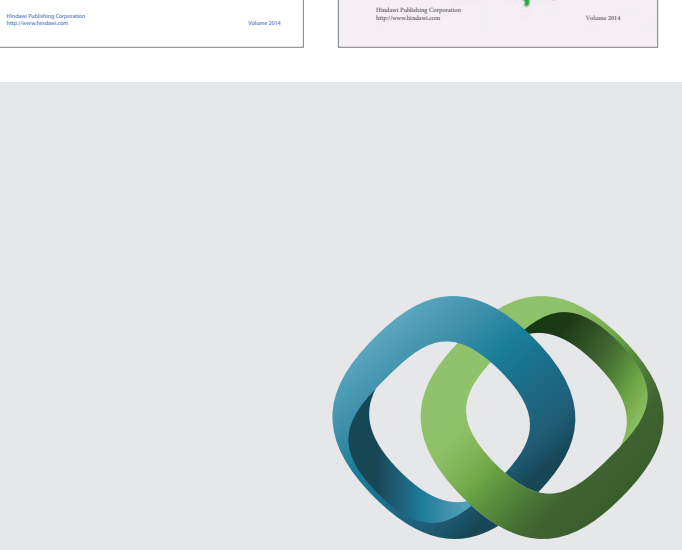

\section{Hindawi}

Submit your manuscripts at

http://www.hindawi.com
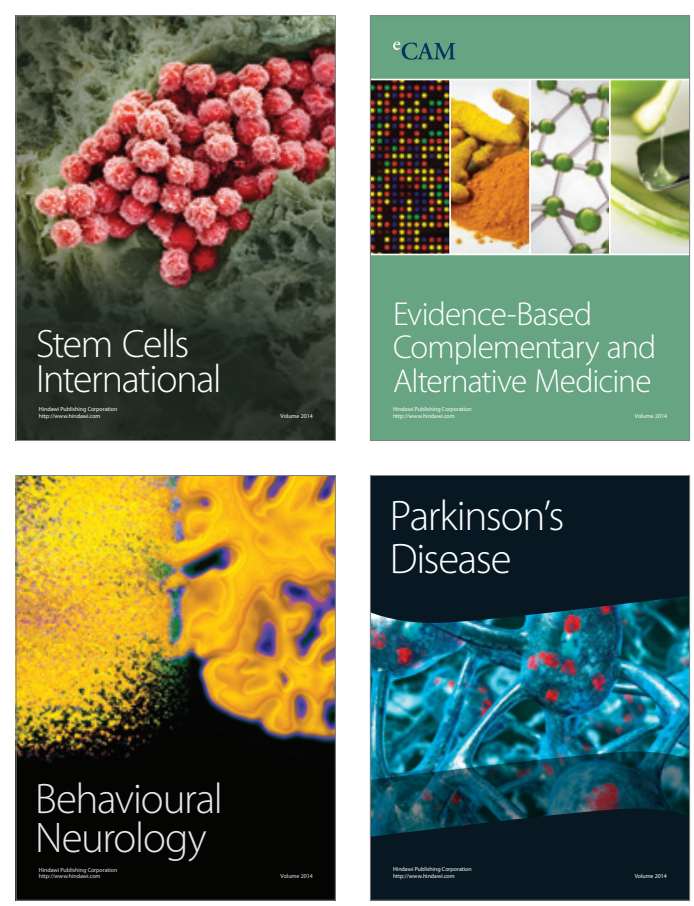

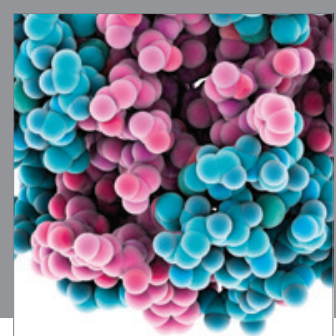

Journal of
Diabetes Research

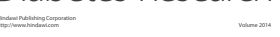

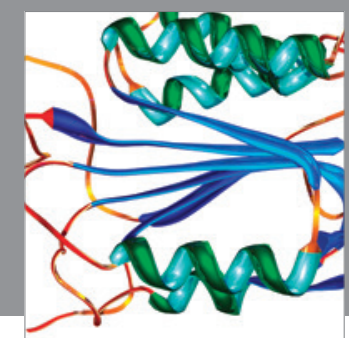

Disease Markers
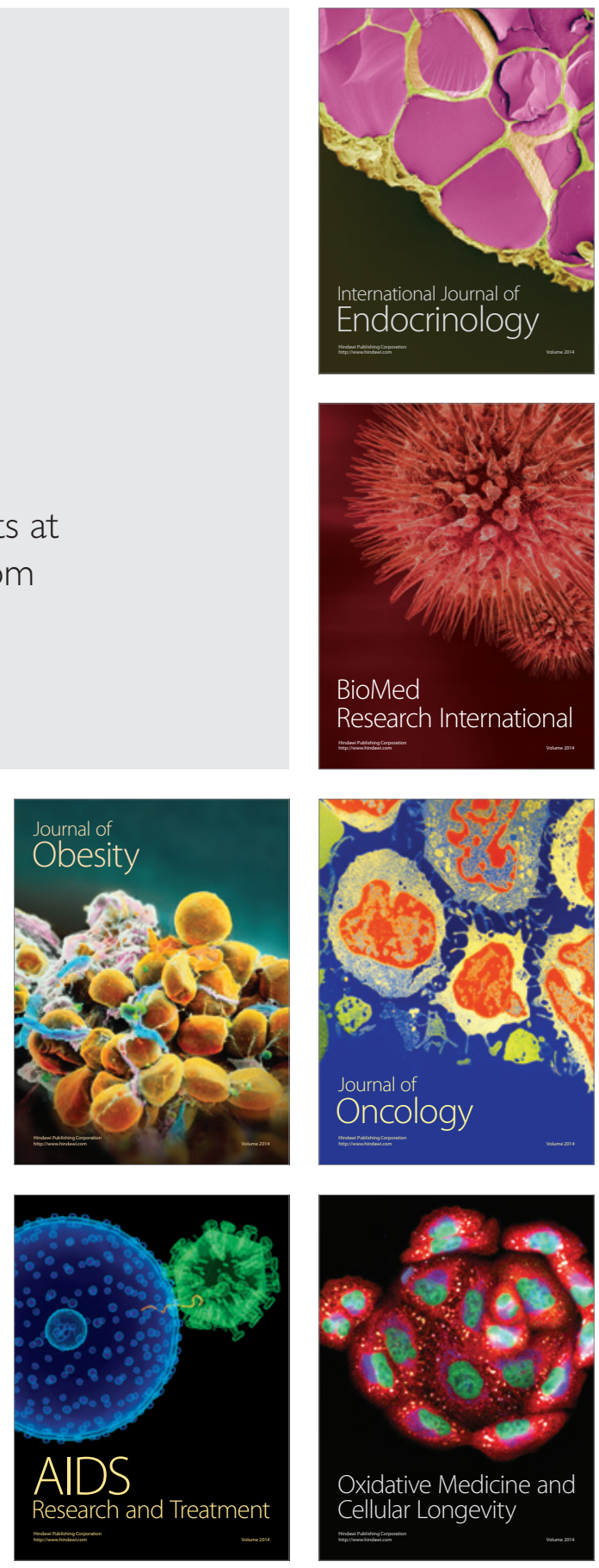